Pacific Journal of Mathematics

ON THE THEOREM OF S. KAKUTANI-M. NAGUMO AND J. L.
WALSH FOR THE MEAN VALUE PROPERTY OF HARMONIC

AND COMPLEX POLYNOMI 


\section{ON THE THEOREM OF S. KAKUTANI-M. NAGUMO AND J. L. WALSH FOR THE MEAN VALUE PROPERTY OF HARMONIC AND COMPLEX POLYNOMIALS}

\section{SHIGERU HARUKI}

Let $K$ be either the field of complex numbers $C$ or the field of real numbers $R$. Let $n$ be a fixed integer $>2$, and $\theta$ denote the number $\exp (2 \pi i / n)$. Let $f, f_{j}: C \rightarrow K$ for $j=$ $0, \cdots, n$. Define $\Lambda_{n}$ and $\Omega_{n}$ by

$$
\begin{aligned}
& \Lambda_{n}(x, y)=n^{-1}\left[\sum_{j=0}^{n-1} f\left(x+\theta^{j} y\right)\right]-f(x), \\
& \Omega_{n}(x, y)=n^{-1}\left[\sum_{j=0}^{n=1} f_{j}\left(x+\theta^{j} y\right)\right]-f_{n}(x),
\end{aligned}
$$

for all $x, y \in C$. Our main result is the following. If $(n+1)$ unknown functions $f_{j}: C \rightarrow K$ for $j=0,1, \cdots, n$ satisfy the quasi mean value property $\Omega_{n}(x, y)=0$ for all $x, y \in C$, then $(n+1)$ unknown functions $f_{j}$ satisfy the difference functional equation $\Delta_{u}^{n} f_{\jmath}(x)=0$ for all $u, x \in C$ and for each $j=0,1, \cdots, n$, where the usual difference operator $A_{u}$ is defined by $\Delta_{u} f(x)=f(x+u)-f(x)$. By using this result we prove somewhat stronger results than the theorem of S. Kakutani-M. Nagumo (Zenkoku, Sūgaku Danwakai, 66 (1935), 10-12) and J. L. Walsh (Bull. Amer. Math. Soc., 42 (1936), 923-930) for the mean value property $\Lambda_{n}(x, y)=0$ of harmonic and complex polynomials.

1. Introduction. Throughout this note $K$ denotes either the field of complex numbers $C$ or the field of real numbers $R$. Let $n$ be a fixed integer $>2$, and $\theta$ denote the number $\exp (2 \pi i / n)$. Let $f, f_{\nu}: C \rightarrow K$ for $\nu=0,1, \cdots, n$. Define $\Lambda_{n}(x, y)$ and $\Omega_{n}(x, y)$ by

$$
\begin{aligned}
& \Lambda_{n}(x, y)=n^{-1}\left[\sum_{\nu=0}^{n-1} f\left(x+\theta^{\nu} y\right)\right]-f(x), \\
& \Omega_{n}(x, y)=n^{-1}\left[\sum_{\nu=0}^{n-1} f_{\nu}\left(x+\theta^{\nu} y\right)\right]-f_{n}(x)
\end{aligned}
$$

for all $x, y \in C$. A function $f: C \rightarrow K$ is said to have the mean value property for polynomials if $f$ satisfies the equation

$$
\Lambda_{n}(x, y)=0 \quad \text { for all } x, y \in C,
$$

while, as a generalization of the mean value property, $n+1$ functions $f_{\nu}: C \rightarrow K$ are said to have the quasi mean value property for polynomials if $f_{\nu}$ satisfy the equation

$$
\Omega_{n}(x, y)=0 \quad \text { for all } x, y \in C .
$$


In $1935 \mathrm{~S}$. Kakutani and M. Nagumo [19], and independently, in $1936 \mathrm{~J}$. L. Walsh [29] proved the following theorems concerning the mean value property of harmonic and complex polynomials.

Theorem A. (Kakutani-Nagumo-Walsh.) If $f: C \rightarrow R$ is continuous, the mean value property $A_{n}(x, y)=0$ holds for all $x, y \in C$ if, and only if, $f(x)$ is a harmonic polynomial of degree at most $n-1$.

THEOREM B. An entire function $f$ satisfies the mean value property $\Lambda_{n}(x, y)=0$ for all $x, y \in C$ if and only if $f$ is given by a complex polynomial of degree at most $n-1$.

The above Theorem $\mathrm{A}$ and Theorem $\mathrm{B}$ are direct or indirect motivations for the generalizations and applications of J. Aczél, H. Haruki, M. A. McKiernan and G. N. Sakovič [2], E. F. Beckenbach and M. Reade [3], [4], A. K. Bose [5], L. Flatto [7], [8], [9], A. Friedman and W. Littman [10], A. Garsia [11], H. Haruki [13], [14], S. Haruki [15], [16], [17], J. H. B. Kemperman and D. Girod [21], M. A. McKiernan [25], M. O. Reade [27]. For more details of functional equations of type $A_{n}(x, y)=0$, see M. A. McKiernan [26], and for the relation to Gauss' mean value theorem, harmonic functions and differential equations, see L. Zalcman [30].

The main purpose of this note is to study some more generalizations of Theorem A and Theorem B from the standpoint of the theory of finite difference functional equations.

2. P-additive symmetrical mappings, generalized polynomials and $\Delta_{y}^{n} f(x)=0$. In this section we present some notation, definitions for $p$-additive symmetrical mappings, generalized polynomials and results of S. Mazur and W. Orlicz [23] for the finite difference functional equation $\Delta_{y}^{n} f(x)=0$.

Definition. A mapping $Q^{p}: C \rightarrow K$ is called a homogeneous polynomial of degree $p$ if and only if there exists a $p$-additive symmetrical mapping $Q_{p}: C^{p} \rightarrow K$; that is, $Q_{p}\left(x_{1}, \cdots, x_{p}\right)=Q_{p}\left(x_{i_{1}}, \cdots, x_{i_{p}}\right)$ for all $\left(x_{1}, \cdots, x_{p}\right) \in C$ and for all permutations $\left(i_{1}, \cdots, i_{p}\right)$ of the sequence $(1, \cdots, p)$ and $Q_{p}$ is an additive function in each $x_{q}, 1 \leqq q \leqq p$, such that $Q^{p}(x)=Q_{p}(x, \cdots, x)$ for all $x \in C$. We say that $Q_{p}$ is associated with $Q^{p}$ or that $Q_{p}$ generates $Q^{p}$.

We agree that for $p=0$ a homogeneous polynomial of degree zero is a constant. If $p$ is a fixed positive integer, then $\pi_{p}: C \rightarrow C^{p}$ will denote the diagonal mapping given by $\pi_{p}(x)=(x, \cdots, x)$. It is clear from the relation $Q^{p}(x)=Q_{p}(x, \cdots, x)$ that $Q^{p}: C \rightarrow K$ is the 
composition of two mappings

$$
C \stackrel{\pi_{p}}{\longrightarrow} C^{p} \stackrel{Q_{p}}{\longrightarrow} K \text { and } Q^{p}=Q_{p} \circ \pi_{p} .
$$

If $Q^{p}: C \rightarrow K$ is a homogeneous polynomial of degree $p$, one obtains $Q^{p}(\lambda x)=\lambda^{p} Q^{p}(x)$ for any rational number $\lambda$. Indeed, the relation $Q^{p}=Q_{p}$ yields $Q^{p}(\lambda x)=Q_{p}(\lambda x, \cdots, \lambda x)=\lambda^{p} Q_{p}(x, \cdots, x)=\lambda^{p} Q^{p}(x)$ for all $x \in C$ and for any rational number $\lambda$.

DEFINITION. Let $\beta$ be any nonnegative integer. If $f: C \rightarrow K$ is a finite sum $f=Q^{0}+Q^{1}+\cdots+Q^{\beta}$ of homogeneous polynomials, then $f$ is called a generalized polynomial of degree at most $\beta$.

For $f: C \rightarrow K$ and for $y \in C$ we define the usual difference operator $\Delta_{y}$ by $\Delta_{y} f(x)=f(x+y)-f(x)$. For $y_{i} \in C, i=1,2, \cdots, n$, we inductively define the $n$th order difference operator $\Delta_{y_{1}}^{n}, \cdots, y_{n}$ by

$$
\Delta_{y_{1} \cdots y_{n}}^{n} f(x)=\left(\Delta_{y_{1} \cdots y_{n-1}}^{n-1}\right) \Delta_{y_{n}} f(x) .
$$

Notice that the ring of operators generated by this family of operators is commutative and distributive.

The following general theorem of S. Mazur and W. Orlicz [23] in the theory of finite difference functional equations plays a fundamental role in our study.

Fundamental theorem. Let $M, N$ be fixed integers $\geqq 0$. Let $X$ be an Abelian additive semigroup with unit element 0 and $l x=$ $x+x+\cdots+x$ for integer $l>0, x \in X$, and let $F$ be an Abelian group and $l y=y+y+\cdots+y$ for integer $l>0, y \in F$. Let $f: X \rightarrow F$. The following three statements are equivalent if $M^{N} \neq 0$ in $F$ :

(a) $\Delta_{y}^{N+1} f(x)=0$ for all $x, y \in X$,

(b) $\Delta_{y_{1} \ldots y_{N+1}}^{N+1} f(x)=0$ for all $x, y_{1}, \cdots, y_{N+1} \in X$,

(c) $f$ is a generalized polynomial of degree at most $N$, that is, $f(x)=Q^{0}+Q^{1}(x)+\cdots+Q^{N}(x)$ for all $x \in X$, where $Q^{p}: X \rightarrow F$ for $p=0,1, \cdots, N$ are homogeneous polynomials.

Note that the above Fundamental theorem clearly holds for the case $X=C$ and $F=K$.

Notation. We denote $Q_{\nu}^{p}(x)=Q_{\nu, p}(x, \cdots, x)$ for $\nu=0,1, \cdots, n$, where $Q_{\nu}^{p}: C \rightarrow K$ are homogeneous polynomials of degree $p$ for $\nu=$ $0,1, \cdots, n$.

Notation. Let $Q_{(n-r, r)}(x ; y)$ denote the value of $Q_{n}\left(x_{1}, \cdots, x_{n}\right)$ for $x_{i}=x, i=1, \cdots, n-r$ and $x_{i}=y, i=n-r+1, \cdots, n$. In par- 
ticular $Q_{(0, n)}(y ; x)=Q_{(n, 0)}(x ; y)=Q^{n}(x)$

3. The quasi mean value property $\Omega_{n}(x, y)=0$. Our first result is the following:

THeORem 3.1. If $n+1$ unknown functions $f_{\nu}: C \rightarrow K$ for $\nu=$ $0,1, \cdots, n$ satisfy the quasi mean value property $\Omega_{n}(x, y)=0$ for all $x, y \in C$, then there exist generalized polynomials of degree at most $n-1$ such that

$$
f_{\nu}(x)=Q_{\nu}^{0}+Q_{\nu}^{1}(x)+\cdots+Q_{\nu}^{n-1}(x)
$$

for all $x \in C$ and for each $\nu=0,1, \cdots, n$.

The proof of Theorem 3.1 is based on the Lemma 3.1 below. Let $G$ and $H$ be additive Abelian groups. Let $S$ be any field and $G, H$ be a unital $S$-modules. Let $f: G \rightarrow H$ satisfy the equation

$$
\sum_{\imath=0}^{n} \gamma_{\imath} f\left(x+\alpha_{i} y\right)=0 \quad \text { for all } x, y \in G,
$$

where $n>2$ is a given integer, $\gamma_{\imath} \neq 0, \alpha_{i} \neq 0\left(=\alpha_{0}\right)$ for $i=0,1, \cdots, n$ are fixed elements in $S$ and $\alpha_{j} \neq \alpha_{k}$ for $j \neq k$. The above equation is a generalization of the difference functional equation (cf. J. Aczél [1], D. ̌̌. Djoković [6], D. Girod and J. H. B. Kemperman [12], M. H. Ingraham [18], J. H. B. Kemperman [20], [22], G. van der Lijn [28], S. Mazur and W. Orlicz [23], M. A. McKiernan [24], [26])

$$
\Delta_{y}^{n} f(x)=0, \quad \text { i.e., } \quad \sum_{i=0}^{n}(-1)^{n-i}\left(\begin{array}{l}
n \\
i
\end{array}\right) f(x+i y)=0
$$

for all $x, y \in G$. More generally we have

Lemma 3.1. Let $f_{i}: G \rightarrow H$ for $i=0,1, \cdots, n$ satisfy the equation

$$
\sum_{i=0}^{n} f_{\imath}\left(x+\alpha_{i} y\right)=0 \quad \text { for all } \quad x, y \in G,
$$

where $\alpha_{i} \neq 0$ for $i=0,1, \cdots, n$ are fixed elements in $S$ and $\alpha_{j} \neq \alpha_{k}$ for $j \neq k$. Then equation (3.1) implies

(3.2) $\Delta_{u}^{n} f_{i}(x)=0 \quad$ for each $i=0,1, \cdots, n$ and for all $x, u \in G$.

Proof of Lemma 3.1. In view of equation (3.1) one can observe the following property.

To eliminate the $k$ th term $f_{k}, 0 \leqq k \leqq n$, we replace $x$ by $x-\alpha_{k} z_{k}$ and $y$ by $y+z_{k}$ in (3.1). 
Indeed, for $k=j$ we have

$$
\begin{gathered}
f_{0}\left(x-\alpha_{j} z_{j}+\alpha_{0} y+\alpha_{0} z_{j}\right)+\cdots+f_{j}\left(x+\alpha_{j} y\right) \\
+\cdots+f_{n}\left(x-\alpha_{j} z_{j}+\alpha_{n} y+\alpha_{n} z_{j}\right)=0
\end{gathered}
$$

for all $x, y, z_{j} \in G$. Take the difference between (3.1) and the above equation to obtain

$$
\Delta_{\left(\alpha_{0}-\alpha_{j} z_{j} j\right.} f_{0}\left(x+\alpha_{0} y\right)+\cdots+0+\cdots+\Delta_{\left(\alpha_{n}-\alpha_{j}\right) z_{j}} f_{n}\left(x+\alpha_{n} y\right)=0
$$

for all $x, y, z_{j} \in G$, since $f_{j}\left(x+\alpha_{j} y\right)$ is unchanged. Thus $f_{j}$ is eliminated. If the same argument (3.3) is repeated $(n-1)$ times, then (3.4) yields

$$
\Delta_{\left(\alpha_{0}-\alpha_{\jmath}\right) z_{j}} \Delta_{\beta_{1} z_{1}} \cdots \Delta_{\beta_{n_{z}} f_{n}} f_{0}\left(x+\alpha_{0} y\right)=0
$$

for all $x, y, z_{1}, \cdots, z_{n} \in G$, where $\beta_{l}=\alpha_{0}-\alpha_{l}$ for $l=1,2, \cdots, n$ and $l \neq j$. In (3.5), replace $x+\alpha_{0} y$ by $x$ and set $u=\left(\alpha_{0}-\alpha_{j}\right) z_{j}=$ $\beta_{1} z_{1}=\cdots=\beta_{n} z_{n}$. Then (3.5) becomes

$$
J_{u}^{n} f_{0}(x)=0 \quad \text { for all } x, u \in G .
$$

It is clear that an obvious modification can be applied for the terms $f_{k}\left(x+\alpha_{k} y\right)$ for $k=1,2, \cdots, n$ to obtain

$$
J_{u}^{n} f_{l k}(x)=0 \quad \text { for each } k=1,2, \cdots, n \text { and for all } x, u \in G .
$$

Thus (3.1) implies (3.2). The Lemma 3.1 is proved.

Proof of Theorem 3.1. Observe that without loss of generality we may assume one of $\alpha_{i}=0$, i.e., $\alpha_{i} \neq 0=\alpha_{n}, i=0,1, \cdots, n-1$, in Lemma 3.1 in order to obtain the same conclusion. The proof now immediately follows from Lemma 3.1 and the Fundamental theorem with $G=X=C$ and $F=S=H=K$.

4. The mean valued property $A_{n}(x, y)=0$. We first determine the general solution of the mean value property under no regularity assumptions. Then we prove somewhat stronger results than that of Theorem A and Theorem B, when some weak regularity assumptions are imposed on $f$.

THEOREM 4.1. A function $f: C \rightarrow K$ satisfies the mean value property $\Lambda_{n}(x, y)=0$ for all $x, y \in C$ if and only if there exists a generalized polynomial of degree at most $n-1$ such that

$$
f(x)=Q^{0}+Q^{1}(x)+\cdots+Q^{n-1}(x) \text { for all } x \in C,
$$

where the homogeneous polynomials $Q^{p}: C \rightarrow K$ for $p=1, \cdots, n-1$ must satisfy the equation 


$$
\sum_{\nu=0}^{n-1} \sum_{\delta=1}^{n-1} \sum_{\sigma=1}^{\delta}\left(\begin{array}{l}
\delta \\
\sigma
\end{array}\right) Q_{(\delta-\sigma, \sigma)}\left(x ; \theta^{\nu} y\right)=0 \quad \text { for all } \quad x, y \in C
$$

Proof of Theorem 4.1. If $f: C \rightarrow K$ satisfies $\Lambda_{n}(x, y)=0$ for all $x, y \in C$, then (4.1) immediately follows from Theorem 3.1. To show the converse, substitute (4.1) into $\Lambda_{n}(x, y)=0$ to obtain

$$
\begin{aligned}
\sum_{\nu=0}^{n-1}\left(Q^{0}\right. & \left.+Q^{1}\left(x+\theta^{\nu} y\right)+\cdots+Q^{n-1}\left(x+\theta^{\nu} y\right)\right) \\
& =n\left(Q^{0}+Q^{1}(x)+\cdots+Q^{n-1}(x)\right),
\end{aligned}
$$

which implies, since $Q^{n-1}\left(x+\theta^{\nu} y\right)=\sum_{o=0}^{n-1}\left(\begin{array}{c}n-1 \\ \sigma\end{array}\right) Q_{(n-1-\sigma, \sigma)}\left(x ; \theta^{\nu} y\right)$,

$$
\begin{aligned}
\sum_{\nu=0}^{n-1}\left(Q^{0}\right. & +Q^{1}(x)+\cdots+Q^{n-1}(x)+Q^{1}\left(\theta^{\nu} y\right)+\sum_{\sigma=1}^{2}\left(\begin{array}{l}
2 \\
\sigma
\end{array}\right) Q_{(2-\sigma, \sigma)}\left(x ; \theta^{\nu} y\right) \\
& \left.+\cdots+\sum_{\sigma=1}^{n-1}\left(\begin{array}{c}
n-\sigma \\
\sigma
\end{array}\right) Q_{(n-1-\sigma, \sigma)}\left(x ; \theta^{\nu} y\right)\right) \\
= & n\left(Q^{0}+Q^{1}(x)+\cdots+Q^{n-1}(x)\right) .
\end{aligned}
$$

But in order for (4.1) to be the general solution of $\Lambda_{n}(x, y)=0$, the homogeneous polynomials $Q^{\delta}, \delta=1,2, \cdots, n-1$, must satisfy equation (4.3). This case occurs only if

$$
\begin{aligned}
& \sum_{\nu=0}^{n-1}\left\{Q^{1}\left(\theta^{\nu} y\right)+\sum_{\sigma=1}^{2}\left(\begin{array}{l}
2 \\
\sigma
\end{array}\right) Q_{(2-\sigma, \sigma)}\left(x ; \theta^{\nu} y\right)+\cdots+\sum_{\sigma=1}^{n-1}\left(\begin{array}{c}
n-1 \\
\sigma
\end{array}\right) Q_{(n-1-\sigma, \sigma)}\left(x ; \theta^{\nu} y\right)\right\} \\
& =0,
\end{aligned}
$$

which yields (4.2). This proves the Theorem 4.1.

THEOREM 4.2. If a function $f: C \rightarrow R$ satisfies $\Lambda_{n}(x, y)=0$ for all $x, y \in C$, then (4.1) holds for all $x \in C$, where $Q^{p}: C \rightarrow R$ for $p=$ $0,1, \cdots, n-1$. Moreover, $f$ is bounded on a set of positive Lebesgue measure if and only if $f$ is given by a harmonic polynomial of degree at most $n-1$.

LemMa 4.1. Let $f: C \rightarrow K$ be a generalized polynomial of degree at most $n-1$ such that

$$
f(x)=Q^{0}+Q^{1}(x)+\cdots+Q^{n-1}(x)
$$

for all $x \in C$, where $Q^{p}: C \rightarrow K, p=0,1, \cdots, n-1$, are homogeneous polynomials. If $f$ is bounded on a set of positive Lebesgue measure, then $Q^{p}$ for $p=0,1, \cdots, n-1$ are continuous everywhere and hence so is $f$. 
Proof of Lemma 4.1. Replace $x$ by $M x$ for each $M=1,2, \cdots, n$. Then

$$
\left[\begin{array}{c}
f(x) \\
f(2 x) \\
\vdots \\
f(n x)
\end{array}\right]=\left[\begin{array}{ccccc}
1 & 1 & 1 & \cdots & 1 \\
1 & 2 & 2^{2} & \cdots & 2^{n-1} \\
\vdots & \vdots & \vdots & & \vdots \\
1 & n & n^{2} & \cdots & n^{n-1}
\end{array}\right]\left[\begin{array}{c}
Q^{0} \\
Q^{1}(x) \\
\vdots \\
Q^{n-1}(x)
\end{array}\right]
$$

We briefly write this as $|F|=|V||Q|$. Observe that $|V|$ is the van der Monde determinant and is not zero. Therefore $Q^{p}, p=0,1, \cdots$, $n-1$, can be determined uniquely in terms of $f(M x)$ for $M=$ $1,2, \cdots, n$. Since $f$ is bounded on a set of positive Lebesque measure, the $Q^{p}(x)$ for $p=0,1, \cdots, n-1$ are bounded on a set of positive Lebesgue measure for all $x$. On the other hand we have the basic identity

$$
Q_{n-1}\left(x_{1}, \cdots, x_{n-1}\right)=(1 /(n-1) !) \Delta_{x_{1}} \cdots \Delta_{x_{n-1}} Q^{n-1}(x)
$$

for all $x, x_{1}, \cdots, x_{n-1}$. The right side is the sum of $2^{N-1}$ terms of the form

$$
\left((-1)^{n-1-q} /((n-1) !)\right) Q^{n-1}\left(x_{i_{1}}+\cdots+x_{i_{q}}\right)
$$

with $x=0$. But we have just proved that $Q^{p}(x)$ is bounded on a set of positive Lebesgue measure for $p=0,1, \cdots, n-1$ and for all $x$. Hence $Q_{p}$ for $p=0,1, \cdots, n-1$ are also bounded on a set of positive Lebesgue measure for all $x_{1}, \cdots, x_{n-1}$. It is well-known (e.g., [20]) that an additive function $f: C \rightarrow K$ which is bounded on a set of positive measure is continuous everywhere. It follows from this theorem that a $p$-additive mapping which is bounded on a set of positive Lebesgue measure is continuous everywhere. Hence, $Q^{p}$ for each $p=0,1, \cdots, n-1$ is continuous everywhere. Equation (4.1) now shows that $f$ is continuous everywhere. This proves the Lemma 4.1.

Proof of Theorem 4.2. This is a consequence of Lemma 4.1 and Theorem A of Kakutani-Nagumo-Walsh.

For the case $K=C$ we have the following:

TheOREM 4.3. If a function $f: C \rightarrow C$ satisfies $\Lambda_{n}(x, y)=0$ for all $x, y \in C$, then (4.1) holds for all $x \in C$. Further, $f$ is bounded on $a$ set of positive Lebesgue measure if and only if $f$ is a complex polynomial of the form

$$
f(x)=\sum_{s=0}^{n-1} a_{0, s} x^{s}+\sum_{r=1}^{n-1} a_{r, r} \bar{x}^{r},
$$


where $\bar{x}$ denotes the conjugate of $x$.

LemmA 4.2. Let $n$ be a given integer $\geqq 1$, and let $Q_{n}: C^{n} \rightarrow C$ be an n-additive symmetrical mapping and continuous everywhere. Then there exist complex constants $a_{0}, a_{1}, \cdots, a_{n}$ such that for all $x_{1}, \cdots, x_{n} \in C$,

$$
Q_{n}\left(x_{1}, \cdots, x_{n}\right)=\sum_{r=0}^{n}\left(a_{r} \sum_{\left(\begin{array}{c}
n \\
r
\end{array}\right)} x_{1} x_{2} \cdots x_{r} \bar{x}_{r+1} \bar{x}_{r+2} \cdots \bar{x}_{n}\right) .
$$

Proof of Lemma 4.2. For $n=1$ we have

$$
Q_{1}\left(x_{1}+x_{2}\right)=Q_{1}\left(x_{1}\right)+Q_{1}\left(x_{2}\right) \quad \text { for all } x_{1}, x_{2} \in C,
$$

whose continuous solutions are well-known (e.g., see J. Azcél [1, p. 217]) to be of the form

$$
Q_{1}(x)=A x+B \bar{x}
$$

where $A$ and $B$ are complex constants. We now assume that (4.5) is true for $n=m \geqq 1$. For $n=m+1$ the continuous solution of the equation

$$
Q_{m+1}\left(x_{1}, \cdots, x_{m}, y+z\right)=Q_{m+1}\left(x_{1}, \cdots, x_{m}, y\right)+Q_{m+1}\left(x_{1}, \cdots, x_{m}, z\right)
$$

for all $x_{1}, \cdots, x_{m}, y, z \in C$ is given by

$$
Q_{m+1}\left(x_{1}, \cdots, x_{m}, x_{m+1}\right)=\sum_{r=0}^{m}\left(A_{r}\left(x_{m+1}\right) \sum_{\left(\begin{array}{c}
m \\
r
\end{array}\right)} x_{1} x_{2} \cdots x_{r} \bar{x}_{r+1} \bar{x}_{r+2} \cdots \bar{x}_{m}\right) .
$$

Substitute (4.7) into (4.6) to obtain

$$
\begin{aligned}
\sum_{r=0}^{m}\left(A_{r}(y+z) \sum_{\left(\begin{array}{c}
m \\
r
\end{array}\right)} x_{1} x_{2} \cdots x_{r} \bar{x}_{r+1} \bar{x}_{r+2} \cdots \bar{x}_{m}\right) \\
=\sum_{r=0}^{m}\left(A_{r}(y) \sum_{\left(\begin{array}{c}
m \\
r
\end{array}\right)} x_{1} x_{2} \cdots x_{r} \bar{x}_{r+1} \bar{x}_{r+2} \cdots \bar{x}_{m}\right) . \\
\quad+\sum_{r=0}^{m}\left(A_{r}(z) \sum_{\left(\begin{array}{c}
m \\
r
\end{array}\right)} x_{1} x_{2} \cdots x_{r} \bar{x}_{r+1} \bar{x}_{r+2} \cdots \bar{x}_{m}\right) .
\end{aligned}
$$

By the uniqueness theorem of polynomial coefficients we have

$$
A_{r}(y+z)=A_{r}(y)+A_{r}(z) \quad \text { for each } \quad r=0,1, \cdots, n
$$

and $A_{r}(x)=\alpha_{r} x+\beta_{r} \bar{x}$ for each $r$, where $\alpha_{r}$ and $\beta_{r}$ are complex constants. This solution in (4.7) implies

$$
Q_{m+1}=\sum_{r=0}^{m}\left(\left(\alpha_{r} x_{m+1}+\beta_{r} \bar{x}_{m+1}\right) \sum_{\left(\begin{array}{c}
m \\
r
\end{array}\right)} x_{1} x_{2} \cdots x_{r} \bar{x}_{r+1} \bar{x}_{r+2} \cdots \bar{x}_{m}\right)
$$


which shows that there exist complex constants $a_{0}, a_{1}, \cdots, a_{m+1}$ such that

$$
Q_{m+1}=\sum_{r=0}^{m+1}\left(a_{r} \sum_{\substack{m+1 \\ r}} x_{1} x_{2} \cdots x_{r} \bar{x}_{r+1} \bar{x}_{r+2} \cdots \bar{x}_{m+1}\right),
$$

yielding the Lemma 4.2.

Note that in particular for the case $x_{1}=x_{2}=\cdots=x_{r}=\bar{x}_{r+1}=$ $\bar{x}_{r+2}=\cdots=\bar{x}_{m}$, (4.5) becomes

$$
Q^{n}(x)=\sum_{r=0}^{n} a_{r} x^{n-r} \bar{x}^{r}
$$

Proof of Theorem 4.3. By applying Lemma 4.1 with $K=C$ we obtain that $Q^{p}$ is continuous for each $p=0,1, \cdots, n-1$. Hence, Lemma 4.2 with (4.8) yields

$$
Q^{p}(x)=\sum_{r=0}^{p} a_{r} x^{p-r} \bar{x}^{r} \quad \text { for each } \quad p=0,1, \cdots, n-1 .
$$

Hence, by (4.1), we have

$$
f(x)=\sum_{s=0}^{n-1} \sum_{r=0}^{s} a_{r, s} x^{s-r} \bar{x}^{r} .
$$

Conversely, if (4.9) is substituted in the mean value property $A_{n}(x, y)=0$, then we obtain

$$
\begin{array}{rl}
\sum_{\nu=0}^{n-1}\left\{\left[a_{0,0}\right]\right. & +\left[a_{0,1}\left(x+\theta^{\nu} y\right)+a_{1,1}\left(\bar{x}+\bar{\theta}^{\nu} \bar{y}\right)\right] \\
& +\left[a_{0,2}\left(x+\theta^{\nu} y\right)^{2}+a_{1,2}\left(x+\theta^{\nu} y\right)\left(\bar{x}+\bar{\theta}^{\nu} \bar{y}\right)+a_{2,2}\left(\bar{x}+\bar{\theta}^{\nu} \bar{y}\right)^{2}\right] \\
& +\cdots+\left[a_{0, n-1}\left(x+\theta^{\nu} y\right)^{n-1}+a_{1, n-1}\left(x+\theta^{\nu} y\right)^{n-2}\left(\bar{x}+\bar{\theta}^{\nu} \bar{y}\right)\right. \\
& \left.\left.+\cdots+a_{n-1, n-1}\left(\bar{x}+\bar{\theta}^{\nu} \bar{y}\right)^{n-1}\right]\right\} \\
=n & n \sum_{s=0}^{n-1} \sum_{r=0}^{s} a_{r, s} x^{s-r} \bar{x}^{r} .
\end{array}
$$

By expanding both sides of (4.10) and comparing coefficients $a_{r, s}$ one observes that (4.9) satisfies the mean value property $\Lambda_{n}(x, y)=0$ if $a_{r, s}=0$ for $r \neq s, r, s=1, \cdots, n-1$, since the right side of (4.10) is independent of $y$ and $\bar{y}$, and

$$
\begin{array}{ll}
\sum_{\nu=0}^{n-1}\left(\theta^{\nu} \bar{\theta}^{\nu}\right)^{p}=n & \text { for } \quad p=0,1, \cdots, n-1, \\
\sum_{\nu=0}^{n-1}\left(\theta^{\nu}\right)^{p}=0 \quad \text { for } \quad p=1, \cdots, n-1, & \text { for } \quad p=1, \cdots, n-1, \\
\sum_{\nu=0}^{n-1}\left(\bar{\theta}^{\nu}\right)^{p}=0 \quad &
\end{array}
$$

and 


$$
\sum_{\nu=0}^{n-1}\left(\theta^{\nu}\right)^{j}\left(\bar{\theta}^{\nu}\right)^{l}=0 \quad \text { for } \quad j \neq l, j, l=1, \cdots, n-1
$$

Therefore, we obtain

$$
f(x)=\sum_{s=0}^{n-1} a_{0, s} x^{s}+\sum_{r=1}^{n-1} a_{r, r} \bar{x}^{r} .
$$

This proves the Theorem 4.3.

The author wishes to thank very much Professor J. Aczél for his advice and encouragement.

\section{REFERENCES}

1. J. Aczél, Lectures on Functional Equations and Their Applications, Academic Press, New York and London, 1966.

2. J. Aczél, H. Haruki, M. A. McKiernan and G. N. Saković, General and regular solutions of functional equations characterizing harmonic polynomials, Aequationes Math., 1 (1968), 37-53.

3. E. F. Beckenbach and M. Reade, Mean values and harmonic polynomials, Trans, Amer. Math. Soc., 53 (1943), 230-238.

4. - Regular solids and harmonic polynomials, Duke Math. J., 12 (1945), 629-644.

5. A. K. Bose, Functions satisfying a weighted average property, Trans, Amer. Math. Soc., 118 (1965), 472-487.

6. D. ̌̌. Djokovie, A representation theorem for $\left(x_{1}-1\right)\left(x_{2}-1\right) \cdots\left(x_{n}-1\right)$ and its ap. plications, Ann. Polon. Math., 22 (1969), 189-198.

7. L. Flatto, Functions with a mean value property, J. Math. Mech., 10 (1961), 11-18.

8. - Functions with a mean value property II, Amer. J. Math., 85 (1963), 248-270.

9. - Basic sets of invariants for finite reflection groups, Bull. Amer. Math. Soc., 74 (1968), 730-734.

10. A. Friedman and W. Littman, Functions satisfying the mean value property, Trans. Amer. Math. Soc., 102 (1962), 167-180.

11. A. Garsia, A note on the mean value property, Trans. Amer. Math. Soc., 102 (1962), 181-186.

12. D. Girod and J. H. B. Kemperman, On the functional equation $\sum_{j=0}^{n} a_{j} f\left(x+T_{j} y\right)=0$, Aequationes Math., 3 (1970), 230.

13. H. Haruki, On an integral mean-value theorem in analytic function theory, J. London Math. Soc., 43 (1968), 551-552.

14. - On a definite integral mean-value problem on a regular polygon (in Japanese), Sûgaku, 22 (1970), 131-134.

15. S. Haruki, On two functional equations connected with a mean-value property of polynomials, Aequationes Math., 6 (1971), 275-277.

16. - Four different unknown functions satisfying the triangle mean value prop. erty for harmonic polynomials, Ann. Polon. Math., 33 (1977), 219-221.

17. - Four different unknown functions satisfying the triangle mean value property for harmonic polynomials II, preprint.

18. M. H. Ingraham, Solution of certain functional equations relative to a general linear set, Trans. Amer. Math. Soc., 28 (1926), 287-300.

19. S. Kakutani and M. Nagumo, About the functional equation $\sum_{\nu=0}^{n=1} f\left(z+e^{(2 \nu \pi / n) i} \xi\right)=$ $n f(z)$ (in Japanese), Zenkoku Sûgaku Danwakai, 66 (1935), 10-12.

20. J. H. B. Kemperman, A general functional equation, Trans, Amer. Math. Soc., 86 (1957), 28-56. 
21. J. H. B. Kemperman and D. Girod, A general solution of the functional equation $\sum_{j=0}^{n} a_{j} f\left(x+T_{j} y\right)=0$, preprint.

22. J. H. B. Kemperman, On a generalized difference property, Aequationes Math., 3 (1970), 231.

23. S. Mazur and W. Orlicz, Grundlegende Eigenschaften der polynomischen Opera. tionen, Studia Mathematica, 5 (1934), 50-68.

24. M. A. McKiernan, On vanishing $n$th ordered differences and Hamel basis, Ann. Polon. Math., 19 (1967), 331-336.

25. - Boundness of a set of positive measure and the mean value property char. acterizes polynomials on a space $V^{n}$, Aequationes Math., 4 (1970), 31-36.

26. - Difference and mean value type functional equations, in functional equa. tions and inequalities, C.I.M.E., III Ciclo, La Mendola 1970. Cremenese, Rome, (1971), 259-286.

27. M. O. Reade, Harmonic polynomials, Duke Math. J., 16 (1949), 627-631.

28. G. van Lijn, La definition des polynômes dans les groups abéliéns, Fund. Math., 33 (1945), 42-50.

29. J. L. Walsh, A mean value theorem for polynomials and harmonic polynomials, Bull. Amer. Math. Soc., 42 (1936), 923-930.

30. L. Zalcman, Mean values and differential equations, Israel J. Math., 14 (1973), 339352 .

Received November 10, 1978. Work supported by NRC Grant A-2972.

UNIVERSITY OF WATERLOO

Waterloo, Ontario

N2L 3G1 CANADA

Current address: Department of Applied Mathematics

Okayama University of Science

Okayama 700, Japan 



\section{PACIFIC JOURNAL OF MATHEMATICS}

\section{EDITORS}

DONALD BABBITT (Managing Editor)

University of Galifornia

Los Angeles, California 90024

Hugo RossI

University of Utah

Salt Lake City, UT 84112

C. C. MOORE AND ANDREW OGG

University of California

Berkeley, CA 94720

\section{J. DugundjI}

Department of Mathematics University of Southern California

Los Angeles, California 90007

R. Finn AND J. Milgram

Stanford University

Stanford, California 94305

\section{ASSOCIATE EDITORS}

R. ARENS

E. F. BECKENBACH

B. H. NeUmanN

F. WOLF

K. YosHIDA

\section{SUPPORTING INSTITUTIONS}

UNIVERSITY OF ARIZONA

UNIVERSITY OF BRITISH COLUMBIA

CALIFORNIA INSTITUTE OF TECHNOLOGY

UNIVERSITY OF CALIFORNIA

MONTANA STATE UNIVERSITY

UNIVERSITY OF NEVADA, RENO

NEW MEXICO STATE UNIVERSITY

OREGON STATE UNIVERSITY
UNIVERSITY OF OREGON

UNIVERSITY OF SOUTHERN CALIFONIA

STANFORD UNIVERSITY

UNIVERSITY OF HAWAII

UNIVERSITY OF TOKYO

UNIVERSITY OF UTAH

WASHINGTON STATE UNIVERSITY

UNIVERSITY OF WASHINGTON 


\section{Pacific Journal of Mathematics}

\section{Vol. 94, No. 1 \\ May, 1981}

Willy Brandal, Conditions for being an FGC domain $\ldots \ldots \ldots \ldots \ldots \ldots \ldots$

Allan Calder and Frank Williams, Incompressibility of maps and the

homotopy invariance of Čech cohomology $\ldots \ldots \ldots \ldots \ldots \ldots \ldots \ldots \ldots$

Jacques Chaumat, Quelques propriétés du couple d'espaces vectoriels

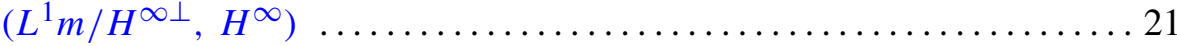

Manfred Droste and Rüdiger Göbel, Products of conjugate permutations . . 47

Jean Esterle, Rates of decrease of sequences of powers in commutative

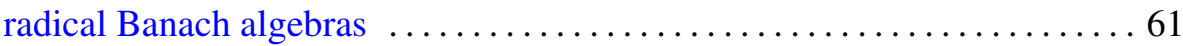

Allan Fryant, Ultraspherical expansions and pseudo analytic functions . . . 83

John Hannah, Homogenization of regular rings of bounded index. II . . . . . 107

Shigeru Haruki, On the theorem of S. Kakutani-M. Nagumo and J. L. Walsh

for the mean value property of harmonic and complex polynomials $\ldots .113$

Hugh M. Hilden, Representations of homology 3-spheres $\ldots \ldots \ldots \ldots \ldots 125$

Craig Huneke, A characterization of locally Macaulay completions . . . . . 131

Takesi Isiwata, Closed ultrafilters and realcompactness ................. 139

Joseph Weston Kitchen, Jr. and David A. Robbins, Tensor products of

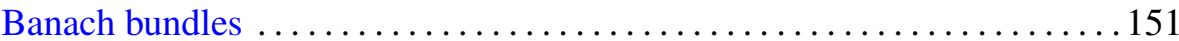

Allan J. Kroopnick, Note on bounded $L^{p}$-solutions of a generalized Liénard

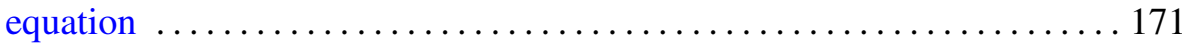

Ajay Kumar and Ajit Kaur Chilana, Spectral synthesis in products and quotients of hypergroups

Charles Livingston, Homology cobordisms of 3-manifolds, knot

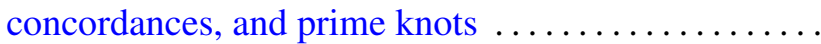

Hans Opolka, Projective representations of finite groups in cyclotomic fields

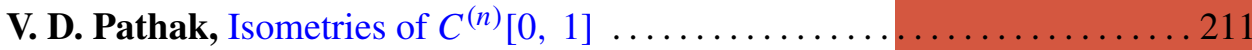

Mark Allan Pinsky, On the spectrum of Cartan-Hadamard manifolds . . . . . 2223

Judith Roitman, The number of automorphisms of an atomic Boolean

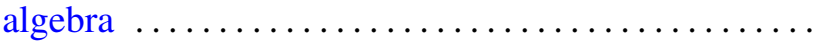

Kai Wang, Locally smooth torus group actions on integral cohomology complex projective spaces 